\title{
Antimicrobials for the treatment of drug- resistant Acinetobacter baumannii pneumonia in critically ill patients: a systemic review and Bayesian network meta-analysis
}

Su Young Jung ${ }^{1,2}$, Seung Hee Lee ${ }^{1}$, Soo Young Lee ${ }^{3,4}$, Seungwon Yang ${ }^{5}$, Hayeon Noh ${ }^{5}$, Eun Kyoung Chung ${ }^{3^{*+}}$ and Jangik I. Lee $e^{1,2^{*}+}$

\begin{abstract}
Background: An optimal therapy for the treatment of pneumonia caused by drug-resistant Acinetobacter baumannii remains unclear. This study aims to compare various antimicrobial strategies and to determine the most effective therapy for pneumonia using a network meta-analysis.

Methods: Systematic search and quality assessment were performed to select eligible studies reporting one of the following outcomes: all-cause mortality, clinical cure, and microbiological eradication. The primary outcome was allcause mortality. A network meta-analysis was conducted with a Bayesian approach. Antimicrobial treatments were ranked based on surface under the cumulative ranking curve (SUCRA) value along with estimated median outcome rate and corresponding 95\% credible intervals (Crls). Two treatments were considered significantly different if a posterior probability of superiority $(P)$ was greater than $97.5 \%$.

Results: Twenty-three studies evaluating 15 antimicrobial treatments were included. Intravenous colistin monotherapy (IV COL) was selected as a common comparator, serving as a bridge for developing the network. Five treatments ranked higher than IV COL (SUCRA, 57.1\%; median all-cause mortality $0.45,95 \%$ Crl 0.41-0.48) for reducing all-cause mortality: sulbactam monotherapy (SUL, 100.0\%; 0.18, 0.04-0.42), high-dose SUL (HD SUL, 85.7\%; 0.31, 0.07-0.71), fosfomycin plus IV COL (FOS + IV COL, 78.6\%; 0.34, 0.19-0.54), inhaled COL plus IV COL (IH COL + IV COL, 71.4\%; 0.39, 0.32-0.46), and high-dose tigecycline (HD TIG, 71.4\%; 0.39, 0.16-0.67). Those five treatments also ranked higher than IV COL (SUCRA, 45.5\%) for improving clinical cure $(72.7 \%, 72.7 \%, 63.6 \%, 81.8 \%$, and 90.9\%, respectively). Among the five treatments, SUL $(P=98.1 \%)$ and $I H C O L+I V C O L ~(P=99.9 \%)$ were significantly superior to IV COL for patient survival and clinical cure, respectively. In terms of microbiological eradication, FOS + IV COL $(P=99.8 \%)$ and SUL $(P=98.9 \%)$ were significantly superior to IV COL.

(Continued on next page)
\end{abstract}

\footnotetext{
* Correspondence: cekchung@khu.ac.kr; jangik.lee@snu.ac.kr

${ }^{\dagger}$ Equal contributors

${ }^{3}$ Department of Pharmacy, College of Pharmacy, Kyung Hee University, 26 Kyungheedae-ro, Dongdaemun-gu, Seoul 02447, Republic of Korea

'Department of Pharmacy, College of Pharmacy, Seoul National University, 1

Gwanak-ro, Gwanak-gu, Seoul 08826, Republic of Korea

Full list of author information is available at the end of the article
} 
(Continued from previous page)

Conclusions: This Bayesian network meta-analysis demonstrated the comparative effectiveness of fifteen antimicrobial treatments for drug-resistant A. baumannii pneumonia in critically ill patients. For survival benefit, SUL appears to be the best treatment followed by HD SUL, FOS + IV COL, IH COL + IV COL, HD TIG, and IV COL therapy, in numerical order.

Keywords: Pneumonia, Critically ill patients, Antimicrobials, Drug-resistant, Acinetobacter baumannii, Network meta-analysis

\section{Background}

Nosocomial pneumonia is a leading cause of death in critically ill patients $[1,2]$. The mortality rates associated with hospital-acquired pneumonia (HAP) or ventilatorassociated pneumonia (VAP) in intensive care units (ICU) range from $38 \%$ to $70 \%$ or higher $[3,4]$. One of the most common pathogens of nosocomial pneumonia is Acinetobacter baumannii (A. baumannii). Because A. baumannii exhibits relatively high virulence and antimicrobial resistance compared with other organisms, the prevalence of multidrug-resistant (MDR) or extensively drug-resistant (XDR) A. baumannii has kept increasing to at least $80 \%$ in past decades [5]. One of the important risk factors for MDR/XDR-bacterial infections in ICU is an inappropriate antibiotic therapy [6]. Considering the paucity of clinical data on the comparative effectiveness of various antimicrobial treatments for MDR/XDR $A$. baumannii pneumonia, it is pressing to accumulate clinically reliable scientific evidence to guide the selection of an optimal antimicrobial treatment for the infection.

Colistin-based, sulbactam-based and tigecycline-based antimicrobial treatments are currently considered the reserved treatment options for MDR/XDR A. baumannii infections [7, 8]. However, the clinical data comparing the antimicrobial treatments adequately with respect to the effectiveness and safety of the treatment of such infections, particularly nosocomial pneumonia, are inconsistent owing to small sample sizes, and substantial between-study heterogeneity [9-13]. In addition, to date there is no consensus based on strong evidence to confirm the therapeutic superiority of a monotherapy or combination therapy and clinical preferences among various antimicrobial combination regimens $[9,10]$. Therefore, clinicians are still facing challenges to apply evidence-based pharmacotherapy in clinical practice for the treatment of nosocomial pneumonia.

Currently available studies have compared the effectiveness of different antimicrobial treatment options only in pairs within the studies [14-16]. Although the results from those studies are informative, the relative effectiveness throughout a variety of therapeutic options remains unknown. Network meta-analysis (NMA) is a relatively novel meta-analysis strategy that integrates both direct evidence from pairwise comparisons within a study and indirect evidence from common-comparator comparisons across the studies $[17,18]$. Compared with conventional meta-analyses, NMA allows comparisons across multiple treatments simultaneously even when the treatments were not directly compared in previous studies. Furthermore, NMA using a Bayesian approach uniquely provides the probability estimates that enable clinicians to make an intuitive pharmacotherapy decision [19]. The Bayesian approach allows us to adopt the strengths from data across multiple studies and does not require an assumption of normal distribution [20]. Therefore, the approach is more favorable than the frequentist approach when small numbers of studies are included in each pair of comparisons.

The aim of this study was to evaluate the comparative effectiveness of currently available antimicrobial options, including monotherapy and combination therapy, for the treatment of critically ill patients with nosocomial pneumonia caused by MDR/XDR A. baumannii, using a Bayesian NMA approach.

\section{Methods}

A systematic review and the Bayesian NMA were performed in accordance with the Preferred Reporting Items for Systematic Reviews and Meta-analyses (PRISMA) extension statement for network metaanalyses [21].

\section{Search strategy}

A comprehensive literature search was performed using the electronic databases of PubMed, Embase, and the Cochrane Central Register of Controlled Trials from the inception of each database to 31 March 2017. The literature search was limited to human studies without language restrictions. In order to identify appropriate articles that evaluated the antimicrobial treatments for patients with nosocomial pneumonia caused by MDR/ XDR pathogens, the following text words and medical subject heading (MeSH) terms were used: "pneumonia", "HAP", or "VAP"; "drug resistant", "carbapenem-resistant", "MDR", or "XDR"; "aminoglycoside", "carbapenems", 
"colistin", "fosfomycin", "glycopeptide", "glycylcycline", "polymyxin", "rifampin", "sulbactam", or "tigecycline". In addition, proceedings from relevant conferences and the references that were listed in all retrieved articles were also manually searched to ensure a complete identification of all eligible studies.

\section{Selection criteria}

The types of study included in the analysis were randomized controlled trials (RCTs) and observational studies in which antimicrobial agents were compared in the treatment of critically ill adult patients with HAP or VAP caused by MDR/XDR A. baumannii. Studies were included if they evaluated at least one of the following three outcomes with clear definitions: all-cause mortality, clinical cure, or microbiological eradication. Allcause mortality, defined as the incidence of deaths from any cause in ICU within the follow-up duration of approximately 30 days, was chosen as the primary outcome variable for the comparison of antimicrobial effectiveness. If all-cause mortality in the ICU was not available in the studies, in-hospital mortality data were used. Clinical cure and microbiological eradication were selected as the secondary outcome variables. Clinical cure was defined as a resolution of signs and symptoms of pneumonia by the end of therapy, whereas microbiological eradication was defined as a confirmed negative result in a follow-up bacterial culture by the end of therapy.

Excluded studies are as follows: (1) case series, (2) studies that enrolled pediatric patients, (3) studies that enrolled patients with community-acquired pneumonia, and (4) studies in which less than half of study population had pneumonia.

\section{Quality assessment and data extraction}

Two investigators (SYJ and SHL) independently screened the titles and abstracts of the articles, and reviewed their full texts based on the pre-specified selection criteria. Any inconsistencies between the two investigators were resolved by extensive discussion with the third investigator (HN). Quality assessment of included studies was performed by two investigators (SYJ and SHL), and was crosschecked by the third investigator (SY). The quality of included RCTs was assessed using the Cochrane Collaboration Risk of Bias Tool [22]. The quality of observational studies was evaluated using the Newcastle-Ottawa Scale (NOS) [23]. Studies with an NOS score $<7$ were considered at high risk of bias [24, 25]. After excluding the studies with high risk of bias, the remaining studies were included in the final analyses. Two investigators (SYJ and SHL) independently extracted the following data from each study using a standardized extraction form: year of publication, type of study design (i.e., RCT or observational study), patient population characteristics, specific antimicrobial therapy with dosing strategy as either an intervention or a comparator, and outcome measurements with their definitions. Antimicrobials administrated at greater than standard dosage for the treatment of MDR/XDR $A$. baumannii pneumonia were separately categorized as high-dose regimens for each antimicrobial agent, as referred to in previous studies [26-28].

\section{Data synthesis and analysis}

Pairwise meta-analyses were initially performed for direct comparisons between different antimicrobial treatments using Stata software (version 13.0, StataCorp, College Station, TX, USA). Homogeneity and consistency were assumed in drawing valid conclusions from NMA analyses [29]. Homogeneity assumption was satisfied when the magnitude of heterogeneity within direct pairwise comparisons was acceptable. Heterogeneity of the treatment effects across trials in each pair was examined by the $Q$ test and quantified using the $I^{2}$ statistic. The $I^{2}$ values $<40 \%, 40 \%-75 \%$, and $>75 \%$ accompanied by a $p$ value $<0.10$ from the $Q$ test were considered mild, moderate, and high heterogeneity, respectively [30]. A consistency assumption referring to the lack of disagreements between direct and indirect comparisons was required to be met in integrating direct and indirect evidence in the NMA [31]. Inconsistency in the entire network of each outcome was assessed by either the Lu-Ades model or design-by-treatment interaction model, according to a configuration of the loop in the network [32-34]. The consistency assumption was rejected when the $p$ value of the inconsistency test was $<0.05$. Publication bias was also evaluated with a funnel plot and Egger's test if ten or more studies were included in each pairwise meta-analysis [30].

The Bayesian NMA was performed for multiple comparisons using WinBUGS (version 1.4.3, MRC Biostatistics Unit, Cambridge, UK). Both fixed-effect and random-effect models were fitted. The best model was chosen based on the deviance information criterion (DIC) that suggests a significantly better fit of the model with a value lowered by $2-3$ points [35]. Markov chain Monte Carlo (MCMC) samplers were run in WinBUGS using three chains with different initial values. Noninformative priors were used to produce posterior distribution for the treatment effects, allowing the data to dominate the final estimates (vague normal distribution with mean of 0 and variance of 0.0001 ). There were 10,000 updates generated for each set of the chains, and the first 10,000 iterations were discarded as the burn-in phase. Brooks-Gelman-Rubin diagnostic plots were used to verify the convergence of the MCMC simulations [36]. 
The estimates of Bayesian NMA were reported as rank probabilities to identify the relative rankings of antimicrobial treatments based on the surface under the cumulative ranking curve (SUCRA), ranging from $0 \%$ (statistically certain to be the worst treatment) to $100 \%$ (statistically certain to be the best treatment) [37]. Because SUCRA rankings could exaggerate the small differences since those are relative values, the estimates of median outcome rates were also reported with the corresponding $95 \%$ credible intervals (CrIs) to specify the absolute magnitude of therapeutic effectiveness. Then, Bayesian posterior probabilities of superiority were calculated to identify a significant difference between an individual treatment and a common comparator. The probabilities of superiority $(P)$ indicated the probabilities of the odds ratio (OR) being $<1$ for all-cause mortality and $>1$ for clinical cure and microbiological eradication. The treatment with $P>97.5 \%$ or $P<2.5 \%$ was considered statistically superior or inferior to the comparator, respectively [38-40]. Although whether or not a confidence interval (CI) for the OR not crosses 1 determines a statistically significant difference in frequentist statistics, the concept of posterior probability in Bayesian statistics was used to demonstrate the certainty of comparative results [41-43].

Sensitivity analyses were performed to evaluate the robustness of the Bayesian NMA results. Although most patients included in this analysis had drugresistant $A$. baumannii pneumonia, the comparative effectiveness of antimicrobial treatments could be affected by type(s) of infection other than pneumonia or causative pathogen(s) other than A. baumannii. Therefore, two sensitivity analyses were performed exclusively using the studies in which all patients had pneumonia or were infected by $A$. baumannii, respectively.

\section{Results}

Study selection and quality assessment

A total of 6688 articles were identified through the electronic database search. Additional 4 records were identified through a manual search by reviewing conference proceedings and the reference lists of retrieved articles. After removing 2017 duplicates, 4675 articles were assessed for relevance by screening the title and abstract. Afterward, 128 relevant articles were screened for eligibility by full-text evaluations. Finally, 23 articles that met the inclusion criteria were included in our Bayesian NMA (Fig. 1). Those 23 articles consist of four RCTs [44-47], three prospective observational studies [48-50], and sixteen retrospective studies [51-66]. A PRISMA extension checklist for reporting systematic reviews comparing multiple treatments involving NMA is shown in Additional file 1: Table S1.

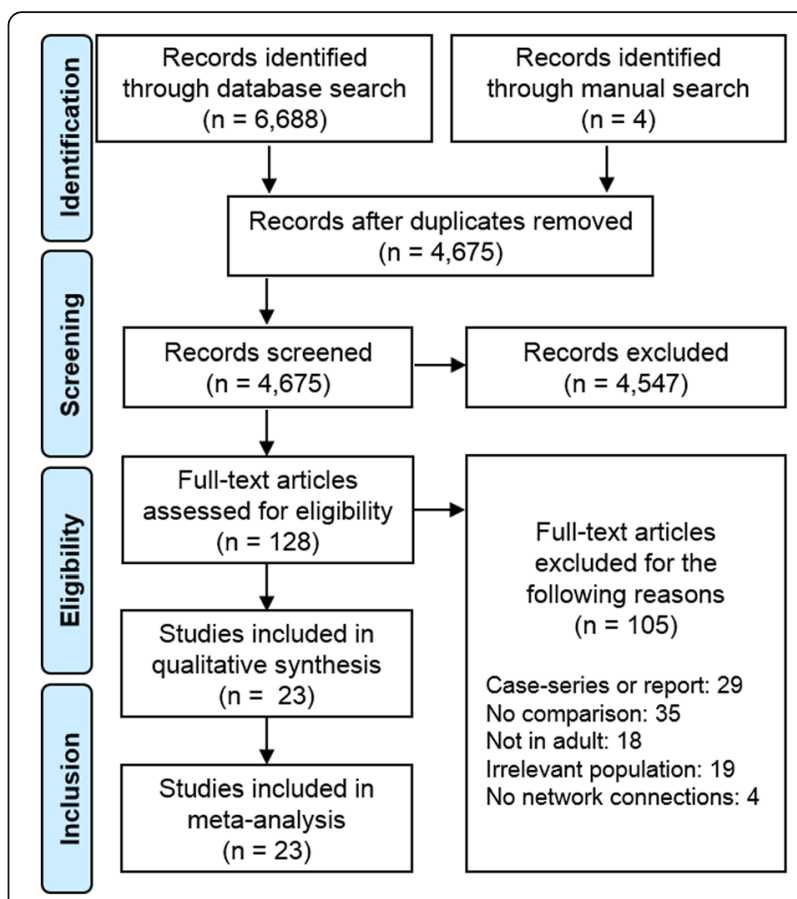

Fig. 1 Study selection process according to the Preferred Reporting Items for Systematic Reviews and Meta-analyses guideline

The results of quality assessment for the included studies are shown in Additional file 2: Table S2 and Table S3. None of the RCTs had high risk of bias for sequence generation, addressing outcome data, selective reporting, or funding source. However, two studies had high risk of bias for double blinding. Bias assessment for allocation concealment was difficult owing to insufficient information available in most studies. All observational studies had a total bias score of at least 7 points based on the NOS, which indicates a low risk of bias.

\section{Study characteristics}

The Bayesian NMA was performed using 23 studies that consist of a total of 2118 adult patients. Table 1 shows the characteristics of the studies including patient population, antimicrobial therapy with a total daily dose, and outcomes of each study. The NMA evaluated 15 different antimicrobial treatments, including intravenous colistin (IV COL), sulbactam (SUL), and tigecycline (TIG) as monotherapy or combination therapy. Among them, SUL and TIG administrated at their higher doses were separately categorized as high-dose (HD) regimens: HD SUL defined as a total daily dose of $9 \mathrm{~g} /$ day or higher and HD TIG as a total daily dose of $200 \mathrm{mg} /$ day after a loading dose of $200 \mathrm{mg}[49,52]$. Across the studies analyzed, the mean (or median) age of the patients ranged from 48 to 82 years, and the mean Acute Physiology and Chronic Health Evaluation II (APACHE II) score from 12 to 24. Most studies reported two-arm 
Table 1 Characteristics of the studies included in the Bayesian network meta-analysis

\begin{tabular}{|c|c|c|c|c|c|c|c|c|}
\hline \multirow{2}{*}{$\begin{array}{l}\text { 1st Author, } \\
\text { publication year }\end{array}$} & \multirow{2}{*}{$\begin{array}{l}\text { Infection } \\
\text { type (\%) }\end{array}$} & \multirow[t]{2}{*}{ Pathogen (\%) } & \multirow{2}{*}{$\begin{array}{l}\text { Antimicrobial therapy } \\
\text { (total daily dose) }\end{array}$} & \multirow{2}{*}{$\begin{array}{l}\text { Mean age } \\
\text { (years) }\end{array}$} & \multirow{2}{*}{$\begin{array}{l}\text { Disease severity } \\
\text { score }^{\text {b }}\end{array}$} & \multicolumn{3}{|c|}{ Outcomes $(n / N)$} \\
\hline & & & & & & $\begin{array}{l}\text { All-cause } \\
\text { mortality }\end{array}$ & $\begin{array}{l}\text { Clinical } \\
\text { cure }\end{array}$ & $\begin{array}{l}\text { Microbiological } \\
\text { eradication }\end{array}$ \\
\hline \multirow[t]{2}{*}{ Abdellatif, 2016 [44] } & \multirow[t]{2}{*}{ VAP 100} & \multirow{2}{*}{$\begin{array}{l}\text { MDR; AB 55, } \\
\text { PA 21, etc. } 24\end{array}$} & CAR (3 g), IV COL (9 MIU) & 53 & SOFA: 6.5 & $18 / 76$ & $55 / 76$ & $57 / 76$ \\
\hline & & & CAR (3 g), IH COL (12 MIU) & 50 & SOFA: 7.0 & 20/73 & $49 / 73$ & $55 / 73$ \\
\hline \multirow[t]{2}{*}{ Amin, 2013 [48] } & \multirow{2}{*}{$\begin{array}{l}\text { VAP (or } \\
\text { HAP) } 100\end{array}$} & \multirow{2}{*}{$\begin{array}{l}\text { MDR; AB 65, } \\
\text { PA 25, KP } 10\end{array}$} & IV COL (3-8 MIU) & 61 & 19.1 & $5 / 12$ & $7 / 12$ & NR \\
\hline & & & $\begin{array}{l}\text { IH COL (3--8 MIU), IV COL } \\
(4 \mathrm{MIU})\end{array}$ & 56 & 18.1 & $8 / 28$ & $22 / 28$ & \\
\hline \multirow[t]{2}{*}{ Aydemir, 2013 [45] } & \multirow[t]{2}{*}{ VAP 100} & \multirow[t]{2}{*}{$C R ; A B 100$} & IV COL (9 MIU) & 63 & 18.0 & $16 / 22$ & $9 / 22$ & $13 / 22$ \\
\hline & & & RIF (600 mg), IV COL (9 MIU) & 58 & 20.1 & $13 / 21$ & $11 / 21$ & $15 / 21$ \\
\hline \multirow[t]{2}{*}{ Betrosian, 2008 [49] } & \multirow[t]{2}{*}{ VAP 100} & \multirow[t]{2}{*}{ MDR; AB 100} & IV COL (9 MIU) & 67 & 14.0 & $5 / 15$ & $9 / 15$ & $10 / 15$ \\
\hline & & & HD SUL (9 g) & 72 & 14.0 & $3 / 13$ & $9 / 13$ & $8 / 13$ \\
\hline \multirow[t]{2}{*}{ Chuang, 2014 [51] } & \multirow{2}{*}{$\begin{array}{l}\text { VAP (or } \\
\text { HAP) } 100\end{array}$} & \multirow[t]{2}{*}{ MDR; AB 100} & IV COL (2.5-5.0 mg/kg) & 64 & 21.6 & $37 / 84$ & NR & NR \\
\hline & & & TIG (100 mg) & 64 & 22.0 & $51 / 84$ & & \\
\hline \multirow{2}{*}{$\begin{array}{l}\text { De Pascale, } \\
2014 \text { [52] }\end{array}$} & \multirow[t]{2}{*}{ VAP 100} & \multirow{2}{*}{$\begin{array}{l}\text { MDR, XDR; AB 44, } \\
\text { KP 48, etc. } 32\end{array}$} & TIG (100 mg) & 65 & SOFA: 7.8 & $20 / 30$ & $10 / 30$ & $7 / 30$ \\
\hline & & & HD TIG (200 mg) & 61 & SOFA: 7.4 & $16 / 33$ & $19 / 33$ & $12 / 33$ \\
\hline Demirdal, 2016 & VAP (or & MDR; AB 100 & IV COL (300 mg) & 63 & NR & $38 / 80$ & $30 / 80$ & $40 / 80$ \\
\hline & & & $\begin{array}{l}\text { IH COL (300 mg), IV COL } \\
(150 \text { mg) }\end{array}$ & 67 & & $23 / 43$ & $16 / 43$ & $20 / 43$ \\
\hline Doshi, 2013 [54] & VAP (or & MDR; $A B$ 64, PA & IV COL (5 mg/kg) & 57 & 24.0 & $19 / 27$ & $20 / 51$ & $11 / 27$ \\
\hline & & & $\begin{array}{l}\text { IH COL (150 or } 300 \text { mg), } \\
\text { IV COL }(5 \mathrm{mg} / \mathrm{kg})\end{array}$ & 61 & 22.4 & $6 / 15$ & $24 / 44$ & $8 / 18$ \\
\hline Durante Mangoni, & VAP (or & XDR; AB 100 & IV COL (6 MIU) & 61 & SAPSII: 39.0 & $45 / 105$ & NR & $47 / 105$ \\
\hline & & & RIF (600 mg), IV COL (6 MIU) & 62 & SAPSII: 40.8 & $45 / 104$ & & $63 / 104$ \\
\hline Frantzeskaki, & VAP 100 & MDR; AB 100 & CAR (6 g), IV COL (9 MIU) & 68 & 12.0 & $5 / 8$ & $6 / 8$ & $6 / 8$ \\
\hline & & & SUL (8 g), IV COL (9 MIU) & 63 & 13.0 & $8 / 16$ & $13 / 16$ & $13 / 16$ \\
\hline Garnacho-Montero, & VAP $86^{d}$ & $C R ; A B 100$ & IV COL (6 or $9 \mathrm{MIU})$ & 63 & 19.0 & $14 / 28$ & $20 / 28$ & $15 / 23$ \\
\hline & & & GLY (TDM), IV COL (6 or 9 MIU) & 54 & 16.0 & $14 / 29$ & $17 / 29$ & $13 / 24$ \\
\hline Hsieh, 2014 [56] & VAP 100 & $X D R ; A B 100$ & IH COL (4 MIU CMS) & 82 & 16.4 & $3 / 9$ & NR & $7 / 9$ \\
\hline & & & $\begin{array}{l}\text { IH COL (4 MIU CMS), } \\
\text { TIG (100 mg) }\end{array}$ & 79 & 17.8 & $10 / 29$ & & $23 / 29$ \\
\hline Kalin, 2012 [57] & VAP 100 & MDR; AB 100 & IV COL (5 or 10 mg/kg) & 48 & $22.0^{f}$ & $7 / 16$ & $6 / 16$ & $11 / 16$ \\
\hline & & & $\begin{array}{l}\text { IH COL }(150 \mathrm{mg}), \text { IV COL } \\
\text { (5 or } 10 \mathrm{mg} / \mathrm{kg})\end{array}$ & 51 & $22.0^{e}$ & $16 / 29$ & $4 / 29$ & $22 / 29$ \\
\hline $\begin{array}{l}\text { Khawcharoenporn, } \\
2014 \text { [58] }\end{array}$ & $\begin{array}{l}\text { VAP } 55, \\
\text { HAP } 45\end{array}$ & $X D R ; A B 100$ & $\begin{array}{l}\text { CAR }(3 \mathrm{~g}), \text { IH COL (160 mg } \\
\text { CMS) }\end{array}$ & $75^{e}$ & $18.0^{e}$ & $18 / 30$ & NR & $16 / 22$ \\
\hline & & & $\begin{array}{l}\text { TIG (100 mg), IH COL (160 mg } \\
\text { CMS) }\end{array}$ & $75^{e}$ & $20.0^{e}$ & $23 / 43$ & & $25 / 38$ \\
\hline & & & $\begin{array}{l}\text { SUL }(6 \mathrm{~g}), \text { IH COL } \\
(160 \mathrm{mg} \text { CMS })\end{array}$ & $75^{e}$ & $18.0^{\mathrm{e}}$ & $60 / 93$ & & $59 / 70$ \\
\hline Kim, 2016 [59] & VAP 73, & MDR or XDR; & IV COL (300 mg) & $67^{e}$ & SOFA: $10.0^{\mathrm{e}}$ & $16 / 40$ & $19 / 40$ & $12 / 40$ \\
\hline & HAF & $A B$ & TIG (100 mg) & $72^{e}$ & SOFA: $9.5^{\mathrm{e}}$ & $14 / 30$ & $14 / 30$ & $7 / 30$ \\
\hline Kofteridis, 2010 [60] & VAP100 & MDR; AB 77, & IV COL (9 MIU) & 62 & 17.7 & $18 / 43$ & $14 / 43$ & $17 / 43$ \\
\hline & & & IH COL (2 MIU), IV COL (9 MIU) & 62 & 17.0 & $10 / 43$ & $23 / 43$ & $19 / 43$ \\
\hline Korbila, 2010 [61] & VAP 100 & MDR; AB 76, & IV COL $(6 \mathrm{MIU})^{f}$ & 61 & 19.2 & $18 / 38$ & $22 / 38$ & NR \\
\hline & & $P f$ & $\begin{array}{l}\text { IH COL }(2 \mathrm{MIU})^{\mathrm{f}}, \mathrm{IV} \mathrm{COL} \\
(7 \mathrm{MIU})^{\mathrm{f}}\end{array}$ & 59 & 17.4 & $24 / 60$ & $46 / 60$ & \\
\hline Kwon, 2014 [62] & $\operatorname{HAP} 75^{9}$ & $X D R ; A B 100$ & IV COL (75-300 mg CMS) & 59 & NR & $17 / 39$ & $19 / 39$ & $18 / 39$ \\
\hline & & & TIG (50-100 mg) & 60 & & $9 / 16$ & $7 / 16$ & $2 / 16$ \\
\hline
\end{tabular}


Table 1 Characteristics of the studies included in the Bayesian network meta-analysis (Continued)

\begin{tabular}{|c|c|c|c|c|c|c|c|c|}
\hline \multirow{2}{*}{$\begin{array}{l}\text { 1st Author, } \\
\text { publication year }\end{array}$} & \multirow{2}{*}{$\begin{array}{l}\text { Infection } \\
\text { type (\%) }\end{array}$} & \multirow[t]{2}{*}{ Pathogen (\%) } & \multirow{2}{*}{$\begin{array}{l}\text { Antimicrobial therapy } \\
\text { (total daily dose) }\end{array}$} & \multirow{2}{*}{$\begin{array}{l}\text { Mean age } \\
\text { (years) }\end{array}$} & \multirow{2}{*}{$\begin{array}{l}\text { Disease severity } \\
\text { score }^{b}\end{array}$} & \multicolumn{3}{|c|}{ Outcomes $(n / N)$} \\
\hline & & & & & & $\begin{array}{l}\text { All-cause } \\
\text { mortality }\end{array}$ & $\begin{array}{l}\text { Clinical } \\
\text { cure }\end{array}$ & $\begin{array}{l}\text { Microbiological } \\
\text { eradication }\end{array}$ \\
\hline \multirow[t]{2}{*}{ Petrosillo, 2014 [63] } & \multirow[t]{2}{*}{ VAP $64^{h}$} & \multirow{2}{*}{$\begin{array}{l}\text { MDR; AB 57, } \\
\text { PA } 18, \text { KP } 17\end{array}$} & IV COL (6 MIU) & $65^{f}$ & $20.0^{\mathrm{e}}$ & $17 / 61$ & NR & NR \\
\hline & & & $\begin{array}{l}\text { GLY (VAN } 2 \text { g; TEl } 400 \text { mg), } \\
\text { IV COL (6 MIU) }\end{array}$ & $68^{f}$ & $21.0^{\mathrm{e}}$ & $14 / 42$ & & \\
\hline \multirow{2}{*}{$\begin{array}{l}\text { Sirijatuphat, } 2014 \\
\text { [47] }\end{array}$} & \multirow{2}{*}{$\begin{array}{l}\text { VAP (or } \\
\text { HAP) } 78^{i}\end{array}$} & \multirow[t]{2}{*}{$C R ; A B 100$} & IV COL (5 mg/kg) & 69 & 21.9 & $27 / 47$ & $26 / 47$ & $38 / 47$ \\
\hline & & & FOS (8 g), IV COL (5 mg/kg) & 67 & 23.0 & $22 / 47$ & $28 / 47$ & $46 / 47$ \\
\hline \multirow{2}{*}{$\begin{array}{l}\text { Tumbarello, } 2013 \\
\text { [64] }\end{array}$} & \multirow[t]{2}{*}{ VAP 100} & \multirow{2}{*}{$\begin{array}{l}\text { XDR; AB 62, } \\
\text { PA 25, KP } 13\end{array}$} & IV COL (0.1 MIU/kg) & $66^{e}$ & SOFA: $8.0^{e}$ & $48 / 104$ & $57 / 104$ & $42 / 84$ \\
\hline & & & $\begin{array}{l}\text { IH COL (3 MIU), IV COL } \\
(0.1 \mathrm{MIU} / \mathrm{kg})\end{array}$ & $64^{\mathrm{e}}$ & SOFA: $7.0^{\mathrm{e}}$ & $45 / 104$ & $72 / 104$ & $52 / 82$ \\
\hline \multirow[t]{3}{*}{ Yilmaz, 2015 [65] } & \multirow[t]{3}{*}{ VAP 100} & \multirow{3}{*}{$\begin{array}{l}\text { MDR or XDR; } \\
\text { AB } 100\end{array}$} & IV COL (225 or 300 mg CMS) & 60 & SAPSII: 43.8 & $7 / 17$ & $13 / 17$ & $9 / 17$ \\
\hline & & & $\begin{array}{l}\text { CAR (IMI } 2 \text { g; MERO } 3 \text { g), IV COL } \\
\text { (225 or } 300 \text { mg CMS) }\end{array}$ & 60 & SAPSII: 50.7 & $16 / 33$ & $21 / 33$ & $21 / 33$ \\
\hline & & & $\begin{array}{l}\text { SUL ( } 3 \text { g), IV COL ( } 225 \text { or } \\
300 \text { mg CMS) }\end{array}$ & 71 & SAPSII: 51.0 & $14 / 20$ & $11 / 20$ & $12 / 20$ \\
\hline \multirow[t]{2}{*}{ Zalts, 2016 [66] } & \multirow[t]{2}{*}{ VAP 100} & \multirow[t]{2}{*}{$C R ; A B 100$} & IV COL (6 MIU) & 57 & 17.8 & $17 / 66$ & $31 / 66$ & $17 / 33$ \\
\hline & & & SUL (4 g) & 50 & 17.2 & $3 / 32$ & $18 / 32$ & $14 / 17$ \\
\hline
\end{tabular}

Abbreviations: AB Acinetobacter baumannii, CAR carbapenem, COL colistin (mostly in colistin base activity (CBA) units), CR carbapenem-resistant, FOS fosfomycin, GLY glycopeptide, HAP hospital-acquired pneumonia, HD high dose, IH inhaled, IV intravenous, IMI imipenem, KP Klebsiella pneumoniae, MDR multidrug-resistant, MERO meropenem, MIU million international units, $n / N$ number of outcome events/number of patients in each intervention, NR not reported, $P A$ Pseudomonas aeruginosa, RIF rifampin, SAPS Simplified Acute Physiology Score, SOFA Sepsis-Related Organ Failure Assessment score, SUL sulbactam (frequently used with ampicillin but described only as SUL in the table), TDM therapeutic drug monitoring, TEI teicoplanin, TIG tigecycline, VAN vancomycin, VAP ventilator-associated pneumonia, XDR extensively drug-resistant

${ }^{\mathrm{a}} \mathrm{COL} 1 \mathrm{MIU} \fallingdotseq 30 \mathrm{mg}$ of colistin base activity (CBA) $\fallingdotseq 80 \mathrm{mg}$ of colistimethate (CMS)

bValues are mean Acute Physiology and Chronic Health Evaluation II score unless otherwise indicated

COther types: bloodstream infection $20 \%$, complicated intra-abdominal infection $2 \%$

dOther types: bloodstream infection $14 \%$

${ }^{\mathrm{e}}$ Median value

${ }^{\mathrm{f}}$ Mean value

${ }^{9}$ Other types: bloodstream infection $13 \%$, wound infection $5 \%$, peritonitis $3 \%$, urinary tract infection $2 \%$, biliary tract infection $2 \%$

hOther types: bloodstream infection $19 \%$, etc. $17 \%$

'Other types: bloodstream infection $5 \%$, urinary tract infection $5 \%$, soft skin tissue infection 3\%, intra-abdominal infection $6 \%$, etc. $3 \%$

comparisons except for two studies with three-arm comparisons. Figure 2 presents the network plots of direct comparisons for each outcome, showing predominant pairwise comparisons of antimicrobials with IV COL. IV COL also served as a bridge node to construct the network with a closed loop, allowing indirect comparisons within the network. Hence, IV COL was selected as a common comparator in the Bayesian NMA.

\section{Assessment of heterogeneity, inconsistency, and model fit}

Table 2 presents the results of heterogeneity in direct pairwise comparisons involving at least two studies. Overall, no statistically significant heterogeneity was observed in most direct pairwise comparisons for each outcome $\left(I^{2}<40 \%, p>0.01\right)$ except for two comparison pairs. Comparison pairs of SUL plus IV COL (SUL + IV COL) versus carbapenem plus IV COL (CAR + IV COR) for all-cause mortality $\left(I^{2}=43.2 \%\right)$, and IV COL versus TIG for microbiological eradication $\left(I^{2}=53.4 \%\right)$ showed moderate heterogeneity, but was not statistically significant $(p>0.10)$. Publication bias was not evaluated because the number of studies included in each pair was fewer than 10. There was no evidence of significant inconsistency in the entire network of each outcome $(p>$ 0.05; Additional file 3: Table S4). Inconsistency was assessed based on the design-by-treatment interaction model because a triangle-loop of each network was formed by one three-arm and one two-arm study in common. All Bayesian NMA estimates were derived from fixed-effect models because of their better fit than that of random-effect models for all three outcomes (allcause mortality, $\mathrm{DIC}_{\text {fixed }}=84$ versus $\mathrm{DIC}_{\text {random }}=86$; clinical cure, 68 versus 70; microbiological eradication, 71 versus 73$)$.

\section{Bayesian NMA ranking}

The SUCRA rank probabilities and Bayesian posterior estimates of the effect of various antimicrobial treatments on all-cause mortality are presented in Fig. 3. SUL monotherapy ranked first for reducing all-cause mortality (SUCRA, 100.0\%; median outcome rate 0.18 , 95\% CrI 0.04-0.42) among the 15 antimicrobial treatments. Four treatments, in addition to SUL, ranked 

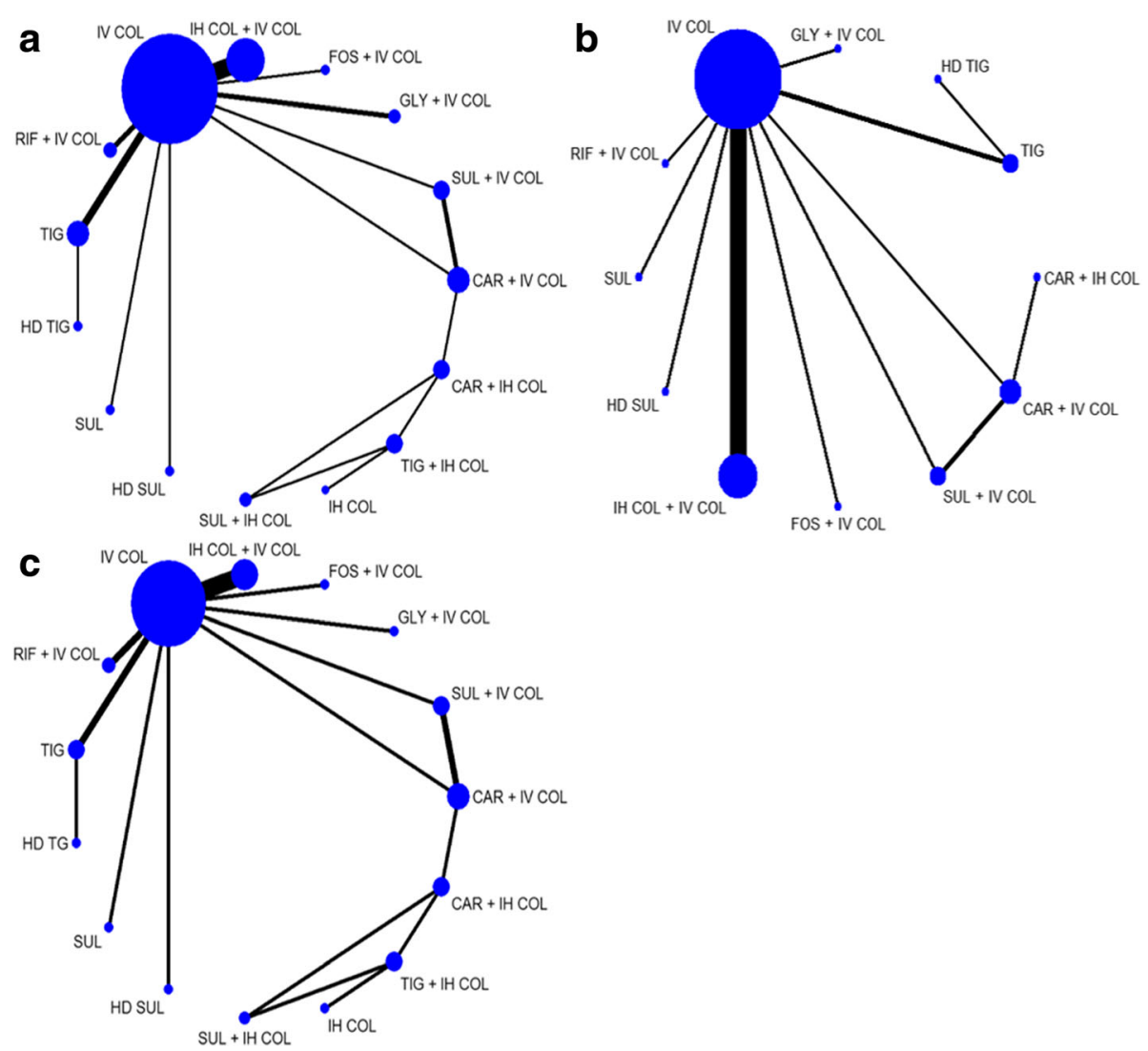

Fig. 2 Networks of direct comparisons. a All-cause mortality. b Clinical cure. c Microbiological eradication. The size of the nodes and the thickness of the lines indicate the sample size and number of trials, respectively. Lines do not connect nodes when there were no head-to-head trials between two treatments. Abbreviations: CAR carbapenem (imipenem or meropenem), COL colistin, FOS fosfomycin, GLY glycopeptide (vancomycin or teicoplanin), HD high-dose, IH inhaled, IV intravenous, RIF rifampin, SUL sulbactam, TIG tigecycline

higher than IV COL (57.1\%; $0.45,0.41-0.48)$ and included HD SUL (85.7\%; 0.31, 0.07-0.71), fosfomycin plus IV COL (FOS + IV COL, 78.6\%; 0.34, 0.19-0.54), inhaled COL plus IV COL (IH COL + IV COL, 71.4\%; $0.39,0.32-0.46)$, and HD TIG (71.4\%; 0.39, 0.16-0.67). Rifampin plus IV COL (RIF + IV COL, 57.1\%; 0.43, $0.31-0.55)$ ranked the same as IV COL. SUL + IV COL (7.1\%; 0.68, 0.37-0.89) ranked the lowest among the 15 different antimicrobial treatments.

Figure 4 shows the SUCRA rank probabilities and Bayesian posterior estimates of the effect of various antimicrobial treatments on clinical cure. HD TIG ranked first for improving clinical cure (SUCRA, 90.9\%; median outcome rate 0.72 , 95\% CrI 0.43-0.91) among the 12 antimicrobial treatments. IV COL (45.5\%; 0.51 , $0.46-0.56)$ and TIG (45.5\%; 0.48, 0.31-0.66) tied for seventh place. Besides HD TIG, IH COL + IV COL (81.8\%; 0.64，0.56-0.70), RIF + IV COL (72.7\%; 0.63, $0.34-0.85)$, HD SUL (72.7\%; 0.62, 0.26-0.90), SUL (72.7\%; 0.60, 0.39-0.79), and FOS + IV COL (63.6\%; $0.56,0.35-0.74)$ ranked higher than IV COL. SUL + IV COL $(0.30,0.09-0.62)$ and CAR + IH COL $(0.29,0.08-$
0.62) were the two lowest-ranked treatments with the same SUCRA value of $9.1 \%$.

Figure 5 presents SUCRA rank probabilities and posterior estimates of the effect of various antimicrobial treatments on microbiological eradication. FOS + IV COL ranked highest for microbiological eradication (SUCRA, 100.0\%; median outcome rate 0.95, 95\% CrI 0.73-1.00) among the 15 different antimicrobial treatments. SUL (92.9\%; 0.85, 0.60-0.97) was next highest, followed by SUL + IH COL (85.7\%; 0.80, 0.40-0.96), and RIF + IV COL (64.3\%; 0.69, 0.57-0.79). CAR + IH COL (57.1\%; 0.66, 0.34-0.89), CAR + IV COL (57.1\%; 0.65, 0.38-0.86), and SUL + IV COL (57.1\%; 0.64, 0.34-0.87) ranked fifth. IH COL + IV COL (42.9\%; 0.60, 0.52-0.69), IH COL (42.9\%; 0.59, 0.10-0.96), and TIG + IH COL (42.9\%; 0.58, 0.19-0.90) tied for eighth place, followed by IV COL (28.6\%; $0.54,0.50-0.59)$. TIG (7.1\%; 0.32 , $0.16-0.52)$ was the lowest-ranked treatment among the 15 different antimicrobial treatments.

Figure 6 displays a clustered SUCRA ranking plot in the three dimensions of $x$-axis (all-cause mortality), $y$ axis (clinical cure), and bubble size (microbiological 
Table 2 Direct pairwise comparisons and heterogeneity

\begin{tabular}{|c|c|c|c|c|c|c|}
\hline \multirow[t]{2}{*}{ Comparison } & \multirow{2}{*}{$\begin{array}{l}\text { Pairwise OR } \\
(95 \% \mathrm{Cl})\end{array}$} & \multirow{2}{*}{$\begin{array}{l}\text { Number of } \\
\text { events }\end{array}$} & \multirow{2}{*}{$\begin{array}{l}\text { Number of } \\
\text { patients }\end{array}$} & \multirow{2}{*}{$\begin{array}{l}\text { Number of } \\
\text { studies }\end{array}$} & \multicolumn{2}{|c|}{ Heterogeneity test } \\
\hline & & & & & $\bar{P}(\%)^{a}$ & $p$ value $^{\mathrm{b}}$ \\
\hline \multicolumn{7}{|l|}{ All-cause mortality } \\
\hline \multicolumn{7}{|l|}{ IV COL vs. } \\
\hline GLY + IV COL & $1.13(0.59-2.19)$ & 59 & 160 & 2 & 0.0 & 0.63 \\
\hline IH COL + IV COL & $0.80(0.58-1.11)$ & 285 & 642 & 7 & 18.2 & 0.29 \\
\hline $\mathrm{RIF}+\mathrm{IV} C \mathrm{CO}$ & $0.94(0.57-1.56)$ & 119 & 252 & 2 & 0.0 & 0.47 \\
\hline $\mathrm{TIG}$ & $1.73(1.08-2.78)$ & 144 & 293 & 3 & 0.0 & 0.78 \\
\hline \multicolumn{7}{|l|}{ CAR + IV COL vs. } \\
\hline SUL + IV COL & $1.59(0.60-4.20)$ & 43 & 77 & 2 & 43.2 & 0.18 \\
\hline \multicolumn{7}{|l|}{ Clinical cure } \\
\hline \multicolumn{7}{|l|}{ IV COL vs. } \\
\hline CAR + IV COL & $0.81(0.37-1.76)$ & 199 & 248 & 2 & 0.0 & 0.46 \\
\hline IH COL + IV COL & $1.60(1.05-2.46)$ & 363 & 695 & 7 & 38.0 & 0.14 \\
\hline $\mathrm{TIG}$ & $0.91(0.43-1.89)$ & 59 & 125 & 2 & 0.0 & 0.83 \\
\hline \multicolumn{7}{|l|}{ CAR + IV COL vs. } \\
\hline SUL + IV COL & $0.60(0.17-2.11)$ & 51 & 77 & 2 & 11.3 & 0.29 \\
\hline \multicolumn{7}{|c|}{ Microbiological eradication } \\
\hline \multicolumn{7}{|l|}{ IV COL vs. } \\
\hline IH COL + IV COL & $1.28(0.88-1.57)$ & 242 & 465 & 5 & 0.0 & 0.73 \\
\hline RIF + IV COL & $1.87(1.13-3.10)$ & 138 & 252 & 2 & 0.0 & 0.90 \\
\hline $\mathrm{TIG}$ & $0.45(0.18-1.11)$ & 39 & 125 & 2 & 53.4 & 0.14 \\
\hline \multicolumn{7}{|l|}{ CAR + IV COL vs. } \\
\hline SUL + IV COL & $1.36(0.45-4.10)$ & 52 & 77 & 2 & 0.0 & 0.95 \\
\hline
\end{tabular}

Abbreviations: CAR carbapenem (imipenem or meropenem), Cl confidence interval, COL colistin, FOS fosfomycin, GLY glycopeptide (vancomycin or teicoplanin), HD high dose, $I H$ inhaled, $I V$ intravenous, OR odds ratio, RIF rifampin, SUL sulbactam, TIG tigecycline, vs. versus

${ }^{a}$ Quantified value of OR variation attributable to heterogeneity

${ }^{b} p$ value from $Q$ test based on chi-square statistic

eradication). The plot includes 12 antimicrobial treatments, commonly included in the analyses of all three outcomes. The relative size of each bubble is in proportion to the $100 \%$ SUCRA value of FOS + IV COL. IV COL fell in the center of the plot with a relatively small bubble size. Five antimicrobial treatments (SUL, HD SUL, FOS + IV COL, IH COL + IV COL, and HD TIG) fell in farther in the right upper corner of the plot than IV COL. SUL was in the farthest-right upper position with a relatively large bubble size among the five treatments, suggesting that overall, SUL is associated with a more favorable therapeutic effect than other treatments for the three outcome variables. The bubble size of both HD SUL and HD TIG was relatively small among the top 5 treatments placed in the farthest-right upper corner, indicating that those treatments have relatively lower benefits in terms of microbiological eradication.

\section{Bayesian posterior probability of superiority}

Table 3 presents the Bayesian probabilities of superiority to IV COL, the key comparator in NMA networks. SUL was significantly superior to IV COL for reducing all-cause mortality (OR 0.27, 95\% CrI $0.06-0.91)$ with a $98.1 \%$ probability of superiority $(P)$. TIG (OR 1.75, 95\% CrI 1.09-2.81) demonstrated a higher all-cause mortality rate than IV COL with $1.0 \%$ of $P$, indicating that TIG is significantly inferior to IV COL $(P<2.5 \%)$ for reducing all-cause mortality. In terms of clinical cure, only IH COL + IV COL was significantly superior to IV COL (OR 1.67, 95\% CrI $1.20-2.29)$ with a $P$ of $99.9 \%$. Except for IH COL + IV $\mathrm{COL}$, there were no statistically significant differences between IV COL and the other 10 antimicrobial treatments that were evaluated in the NMA for the clinical cure outcome. FOS + IV COL (OR 15.20, 95\% CrI 2.27-428.60), RIF + IV COL (1.88, 1.14-3.13), and SUL $(4.82,1.22-25.83)$ were significantly superior to IV COL in terms of microbiological eradication rate (Table 3; $P=99.8 \%, 99.4 \%$, and $98.9 \%$, respectively). On the other hand, TIG was significantly inferior to IV COL $(P=1.7 \%$; OR $0.40,95 \%$ CrI $0.16-0.94)$ in microbiological eradication. 


\begin{tabular}{lcl}
\hline Intervention & $\begin{array}{c}\text { SUCRA } \\
\%\end{array}$ & $\begin{array}{l}\text { Posterior estimates } \\
\text { Median }(95 \% \text { Crl) }\end{array}$ \\
\hline SUL & 100.0 & $0.18(0.04-0.42)$ \\
$\mathrm{HD} \mathrm{SUL}$ & 85.7 & $0.31(0.07-0.71)$ \\
$\mathrm{FOS}+\mathrm{IV} \mathrm{COL}$ & 78.6 & $0.34(0.19-0.54)$ \\
$\mathrm{IH} \mathrm{COL}+\mathrm{IV} \mathrm{COL}$ & 71.4 & $0.39(0.32-0.46)$ \\
$\mathrm{HD}$ TIG & 71.4 & $0.39(0.16-0.67)$ \\
$\mathrm{RIF}+\mathrm{IV} \mathrm{COL}$ & 57.1 & $0.43(0.31-0.55)$ \\
$\mathrm{IV} \mathrm{COL}$ & 57.1 & $0.45(0.41-0.48)$ \\
$\mathrm{GLY}+\mathrm{IV} \mathrm{COL}$ & 50.0 & $0.48(0.32-0.64)$ \\
$\mathrm{IH} \mathrm{COL}$ & 42.9 & $0.52(0.09-0.92)$ \\
$\mathrm{TIG}+\mathrm{IH} \mathrm{COL}$ & 35.7 & $0.54(0.18-0.87)$ \\
$\mathrm{CAR}+\mathrm{IV} \mathrm{COL}$ & 35.7 & $0.56(0.29-0.81)$ \\
$\mathrm{TIG}$ & 28.6 & $0.59(0.47-0.69)$ \\
$\mathrm{CAR}+\mathrm{IH} \mathrm{COL}$ & 21.4 & $0.61(0.28-0.86)$ \\
SUL + IH COL & 14.3 & $0.65(0.26-0.91)$ \\
SUL + IV COL & 7.1 & $0.68(0.37-0.89)$ \\
\hline & &
\end{tabular}

Fig. 3 Surface under the cumulative ranking curve (SUCRA) rankings and posterior estimates of treatment effect on all-cause mortality. Greater SUCRA value indicate higher probability of being the best treatment for reducing all-cause mortality. Abbreviations: CAR carbapenem (imipenem or meropenem), COL colistin, Crl credible interval, FOS fosfomycin, GLY glycopeptide (vancomycin or teicoplanin), HD high-dose, IH inhaled, IV intravenous, RIF rifampin, SUL sulbactam, TIG tigecycline

\begin{tabular}{lcll}
\hline Intervention & $\begin{array}{c}\text { SUCRA } \\
\%\end{array}$ & $\begin{array}{c}\text { Posterior estimates } \\
\text { Median }(95 \% \text { Crl) }\end{array}$ \\
\hline $\mathrm{HD} \mathrm{TIG}$ & 90.9 & $0.72(0.43-0.91)$ \\
$\mathrm{IH} \mathrm{COL}+\mathrm{IV} \mathrm{COL}$ & 81.8 & $0.64(0.56-0.70)$ \\
$\mathrm{RIF}+\mathrm{IV} \mathrm{COL}$ & 72.7 & $0.63(0.34-0.85)$ \\
$\mathrm{HD} \mathrm{SUL}$ & 72.7 & $0.62(0.26-0.90)$ \\
$\mathrm{SUL}$ & 72.7 & $0.60(0.39-0.79)$ \\
$\mathrm{FOS}+\mathrm{IV} \mathrm{COL}$ & 63.6 & $0.56(0.35-0.74)$ \\
$\mathrm{IV} \mathrm{COL}$ & 45.5 & $0.51(0.46-0.56)$ \\
$\mathrm{TIG}$ & 45.5 & $0.48(0.31-0.66)$ \\
$\mathrm{GLY}+\mathrm{IV} \mathrm{COL}$ & 27.3 & $0.37(0.16-0.63)$ \\
$\mathrm{CAR}+\mathrm{IV} \mathrm{COL}$ & 18.2 & $0.34(0.12-0.64)$ \\
$\mathrm{SUL}+\mathrm{IV} \mathrm{COL}$ & 9.1 & $0.30(0.09-0.62)$ \\
$\mathrm{CAR}+\mathrm{IH} \mathrm{COL}$ & 9.1 & $0.29(0.08-0.62)$
\end{tabular}

Fig. 4 Surface under the cumulative ranking curve (SUCRA) rankings and posterior estimates of treatment effect on clinical cure. Greater SUCRA value indicates higher probability of being the best treatment for improving clinical cure. Abbreviations: CAR carbapenem (imipenem or meropenem), COL colistin, Crl credible interval, FOS fosfomycin, GLY glycopeptide (vancomycin or teicoplanin), HD high-dose, IH inhaled, IV intravenous, RIF rifampin, SUL sulbactam, TIG tigecycline 


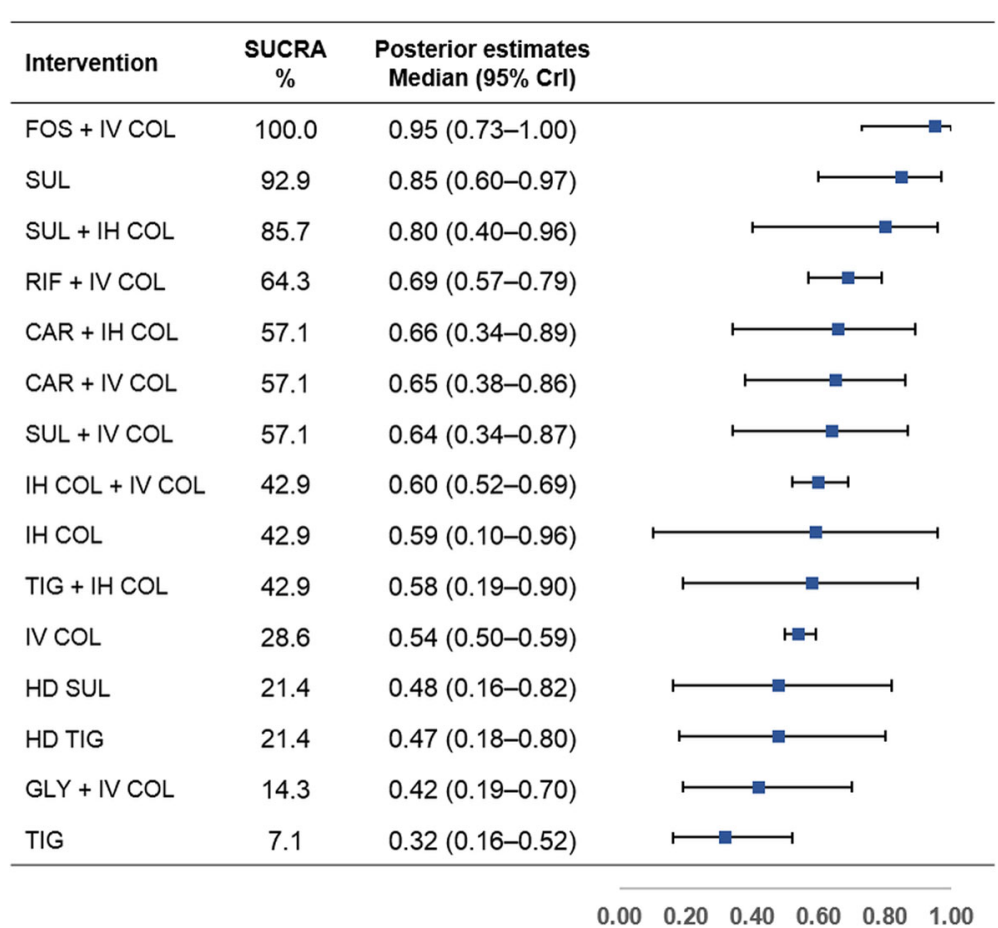

Fig. 5 Surface under the cumulative ranking curve (SUCRA) rankings and posterior estimates of treatment effect on microbiological eradication. Greater SUCRA value indicates higher probability of being the best treatment for improving microbiological eradication. Abbreviations: CAR carbapenem (imipenem or meropenem), COL colistin, Crl credible interval, FOS fosfomycin, GLY glycopeptide (vancomycin or teicoplanin), HD high-dose, IH inhaled, IV intravenous, RIF rifampin, SUL sulbactam, TIG tigecycline

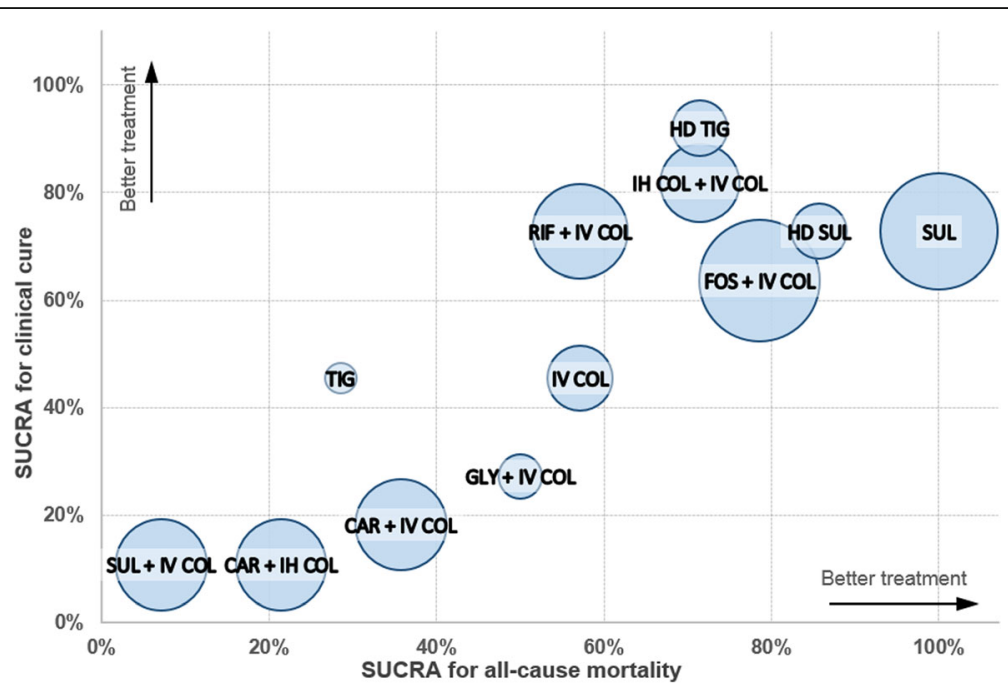

Fig. 6 Clustered ranking plot based on surface under the cumulative ranking curve (SUCRA). The plot shows SUCRA values of twelve antimicrobial treatments, commonly included in the analyses of the following outcomes: all-cause mortality, clinical cure, and microbiological eradication. A treatment lying in the farther-right upper corner is more effective in both all-cause mortality and clinical cure than other treatments. In addition, the larger bubble size reflects the greater SUCRA value in terms of microbiological eradication. Abbreviations: CAR carbapenem (imipenem or meropenem), COL colistin, FOS fosfomycin, GLY glycopeptide (vancomycin or teicoplanin), HD high-dose, IH inhaled, IV intravenous, RIF rifampin, SUL sulbactam, TIG tigecycline 
Table 3 Bayesian NMA estimates of probability of superiority $(P)$ and odds ratio (OR)

\begin{tabular}{|c|c|c|c|c|c|c|}
\hline \multirow[t]{2}{*}{ Treatment } & \multicolumn{2}{|c|}{ All-cause mortality } & \multicolumn{2}{|c|}{ Clinical cure } & \multicolumn{2}{|c|}{ Microbiological eradication } \\
\hline & $p \%$ & OR $(95 \% \mathrm{Crl})$ & $p \%$ & OR (95\% Crl) & $P \%$ & OR $(95 \% \mathrm{Crl})$ \\
\hline$\overline{S U L}$ & $98.1^{*}$ & $0.27(0.06-0.91)$ & 80.4 & $1.45(0.62-3.48)$ & $98.9^{*}$ & $4.82(1.22-25.83)$ \\
\hline $\mathrm{IH}$ COL + IV COL & 92.1 & $0.80(0.58-1.10)$ & $99.9^{*}$ & $1.67(1.20-2.29)$ & 90.4 & $1.28(0.88-1.88)$ \\
\hline FOS + IV COL & 85.3 & $0.64(0.28-1.45)$ & 66.3 & $1.20(0.52-2.72)$ & $99.8^{*}$ & $15.2(2.27-428.6)$ \\
\hline HD SUL & 74.5 & $0.56(0.09-3.17)$ & 70.1 & $1.54(0.32-8.63)$ & 38.3 & $0.79(0.15-3.99)$ \\
\hline HD TIG & 65.1 & $0.81(0.25-2.53)$ & 91.8 & $2.48(0.70-8.89)$ & 36.4 & $0.77(0.19-3.30)$ \\
\hline $\mathrm{RIF}+\mathrm{IV} C \mathrm{CO}$ & 59.7 & $0.94(0.56-1.54)$ & 78.1 & $1.63(0.48-5.54)$ & $99.4^{*}$ & $1.88(1.14-3.13)$ \\
\hline IH COL & 39.8 & $1.33(0.12-13.87)$ & NA & NA & 55.9 & $1.22(0.09-19.93)$ \\
\hline GLY + IV COL & 35.3 & $1.13(0.58-2.20)$ & 14.9 & $0.55(0.17-1.65)$ & 21.6 & $0.62(0.18-2.04)$ \\
\hline $\mathrm{TIG}+\mathrm{IHCOL}$ & 31.3 & $1.47(0.26-8.32)$ & NA & NA & 56.9 & $1.18(0.18-7.40)$ \\
\hline$C A R+I V C O L$ & 21.1 & $1.58(0.49-5.52)$ & 14.2 & $0.50(0.12-1.78)$ & 78.1 & $1.57(0.50-5.45)$ \\
\hline $\mathrm{CAR}+\mathrm{IHCOL}$ & 16.7 & $1.94(0.48-8.19)$ & 9.9 & $0.38(0.08-1.64)$ & 76.5 & $1.65(0.42-7.06)$ \\
\hline $\mathrm{SUL}+\mathrm{IHCOL}$ & 14.2 & $2.32(0.43-12.63)$ & NA & NA & 90.4 & $3.37(0.54-20.94)$ \\
\hline SUL + IV COL & 7.0 & $2.58(0.71-9.88)$ & 10.1 & $0.40(0.09-1.60)$ & 73.3 & $1.51(0.42-5.75)$ \\
\hline $\mathrm{TIG}$ & $1.0^{*}$ & $1.75(1.09-2.81)$ & 38.7 & $0.89(0.43-1.88)$ & $1.7^{*}$ & $0.40(0.16-0.94)$ \\
\hline
\end{tabular}

Abbreviations: CAR carbapenem (imipenem or meropenem), COL colistin, Crl credible interval, FOS fosfomycin, GLY glycopeptide (vancomycin or teicoplanin), HD high-dose, IH inhaled, IV intravenous, NA not available, RIF rifampin, SUL sulbactam, TIG tigecycline

${ }^{*} P>97.5 \%$ and $P<2.5 \%$, statistically significant superiority and inferiority, respectively

\section{Sensitivity analyses}

According to the sensitivity analyses (Additional file 4: Table S5), the orders based on SUCRA values and statistical significance remained similar after removing specific studies from the analysis pool. For the first sensitivity analysis, six studies were eliminated from the original study pool because patients with other infectious diseases besides pneumonia were enrolled in those studies, though the proportion was small. Even after removing those six studies, the SUCRA rankings were comparable with those in the main analysis. In another sensitivity analysis exclusively using 14 studies in which all patients were infected by A. baumannii, the SUCRA value for $\mathrm{IH} C \mathrm{COL}+\mathrm{IV}$ COL therapy was lower, especially for clinical cure, because most of the studies comparing $\mathrm{IH} \mathrm{COL}+\mathrm{IV}$ COL with IV COL were excluded. This sensitivity analysis suggests the robustness of our analysis by showing that concurrent infection site(s) other than the respiratory tract and the type of causative pathogen may not markedly affect the comparative effectiveness estimates of the antimicrobial treatments evaluated in this NMA.

\section{Discussion}

To the best of our knowledge, this is the first and the most comprehensive Bayesian NMA evaluating the comparative effectiveness of various antimicrobial treatment regimens for MDR/XDR A. baumannii pneumonia in critically ill patients. An important finding of our study is that SUL was the most effective therapy to reduce the all-cause mortality in critically ill patients. The top five treatments with high probabilities of survival benefit were SUL (SUCRA 100.0\%), HD SUL (85.7\%), FOS + IV COL (78.6\%), IH COL + IV COL (71.4\%), and HD TIG (71.4\%) (Fig. 3). Those five treatment options generally ranked high for improving clinical cure and microbiological eradication as well. However, HD TIG and HD SUL had relatively lower SUCRA values for microbiological eradication among the 15 different antimicrobial treatments. A possible explanation for those lower rankings in microbiological eradication is that, based on the published data, A. baumannii isolates in the two HD treatment groups were less susceptible to TIG or SUL (MIC $>1 \mathrm{mg} / \mathrm{L}$ for TIG; MIC $>16 \mathrm{mg} / \mathrm{L}$ for SUL, respectively) $[49,52]$.

The results of this study corroborate growing recent evidence that suggests SUL as a promising treatment option in the management of Acinetobacter infections [67-69]. Multiple clinical studies have reported that the patient group treated with SUL had a substantially low rate of mortality, ranging from $17 \%$ to $33 \%$ during approximately 2 weeks of treatment [67-70]. In addition, Oliveira et al. report that polymyxin (colistin or polymyxin B) treatment is significantly associated with higher mortality than SUL, with a relative risk of 1.52 [70]. Similarly, this Bayesian NMA demonstrated that SUL was superior to IV COL with the probabilities of superiority greater than $97.5 \%$ in terms of reducing allcause mortality and improving microbiological eradication. However, caution needs to be exercised when interpreting and applying our findings to clinical practice, owing to the retrospective nature and relatively 
small sample sizes of the studies included in this NMA, and potential inherent bias, if any, in reporting the results of the original studies.

According to a recent conference report from the European Society of Intensive Care Medicine, a 4-hour infusion of SUL 3-4 g every 8 hours is recommended for severe $A$. baumannii infections involving isolates with higher MICs for SUL ( $\geq 8 \mathrm{mg} / \mathrm{L})$ [10]. A recent pharmacodynamic modeling study conducted in healthy adults demonstrated that a 4-hour infusion of SUL $3 \mathrm{~g}$ every 8 hours would be an appropriate dosage regimen of SUL for less-susceptible A. baumannii [71]. The findings from healthy adults may not be generalizable to critically ill patients owing to pharmacokinetic alterations associated with critical illness [71, 72]. Nevertheless, it is noteworthy that prolonged-infusion dosing was found to be a much more effective strategy to achieve a high probability of target concentration attainment over a range of MICs than a dose-escalation strategy [71]. In the HD SUL treatment group in this NMA, the infusion time of HD SUL was within 1 hour even though SUL MIC for isolated A. baumannii was $>16 \mathrm{mg} / \mathrm{L}$ [49]. It could be inferred that a more prolonged infusion time was necessary for improving microbiological eradication, considering the time-dependent antimicrobial activity of SUL [72].

Among combination regimens evaluated in this NMA, FOS + IV COL and IH COL + IV COL had a more beneficial effect on all-cause mortality, with favorable effectiveness in clinical cure and microbiological eradication (Fig. 6). In terms of microbiological eradication, FOS + IV COL demonstrated the greatest SUCRA value in our Bayesian NMA. FOS may be an effective adjunctive therapy for pneumonia caused by MDR/XDR A. baumannii, considering the synergistic effect of COL and FOS in vitro [73-75]. However, owing to the paucity of clinical data evaluating the efficacy and safety of FOS + IV COL, adjunctive FOS should be used with caution until clinical studies adequately confirm its promising effect in patients with severe pneumonia. According to the guideline recently updated by the Infectious Diseases Society of America, adjunct IH COL therapy is suggested for the treatment of HAP/VAP due to A. baumannii that is sensitive only to COL, particularly for patients with insufficient response to IV COL monotherapy [76]. Similarly, in this NMA, the probability of superiority analysis showed that IH COL + IV COL yielded additional therapeutic superiority in terms of clinical cure, and comparable effectiveness in other outcomes, compared with IV COL.

Besides IH COL, several aerosolized antimicrobial agents including amikacin, tobramycin, and fosfomycin were evaluated for the treatment of gram-negative pneumonia in previous studies comparing the efficacy of $\mathrm{IH}$ antimicrobial adjunct therapy to various IV antimicrobial agents with that of IV monotherapy or IH placebo [77-80]. However, antimicrobial agents evaluated in those studies could not be included as treatment nodes in our NMA to construct a single connected network. Furthermore, non-MDR/XDR A. baumannii pneumonia patients were largely included in those studies. Of note, more evidence is required to determine the appropriate administration method of $\mathrm{IH}$ antimicrobials for optimal clinical benefit in patients with pneumonia because the delivery devices, dosing, ventilator settings, and endotracheal tube size may affect the therapeutic response [76, 81].

There are a few limitations in this meta-analysis. The major limitation of this NMA is the absence of aerosolized antimicrobials other than $\mathrm{IH}$ COL as aforementioned. Another important limitation is that the analysis was mostly based on retrospective studies (16 out of 23 studies in total). Considering the retrospective nature of most included studies, this NMA should be viewed as a hypothesis-generating study rather than definitive clinical evidence, despite rigorous quality assessments. The safety profiles of all antimicrobial treatments were not evaluated due to insufficient data on adverse drug reactions and substantial differences between studies in the baseline laboratory values or organ function parameters. In addition, the novel antimicrobial treatments with potential activity against A. baumannii, such as vabomere, plazomicin, cefiderocol and eravacycline, were not included in this NMA owing to absence of published data on the patients with MDR/ XDR A. baumannii pneumonia [82-84]. It remains to be determined whether any of these agents may have a role in this clinically important infection. Additionally, this NMA could not clearly address the role of combination therapy over single-agent therapy. In fact, the purpose of this NMA was not to specifically compare the effectiveness between combination therapy and monotherapy but to evaluate the overall comparative effectiveness of different antimicrobial treatment options. Last, variability in identifying the causative pathogen among the included studies could not completely distinguish colonization from infection in HAP and VAP. Clinical studies more robustly detecting the true infectious organism in HAP or VAP may be necessary to accurately and precisely determine the effects of antimicrobial treatments for MDR/XDR $A$. baumannii pneumonia.

\section{Conclusions}

This Bayesian NMA provides clinically meaningful evidence to aid clinicians in selecting the optimal antimicrobial regimen for the treatment of critically ill patients with MDR/XDR A. baumannii pneumonia. SUL appears to be the best treatment among fifteen different antimicrobial regimens investigated for survival benefit. 
Apart from SUL, FOS + IV COL, HD SUL, IH COL + IV COL, and HD TIG therapy may be appropriate alternative treatment options. The comparative results of our analysis should be tailored to the antimicrobial susceptibility testing result of each individual patient when making treatment decisions. Appropriate and wellcontrolled studies would be necessary to prospectively verify the findings of our current study in patients with nosocomial pneumonia.

\section{Additional files}

Additional file 1: Table S1. PRISMA checklist of items to include when reporting a systematic review and a network meta-analysis. (DOCX 20 kb)

Additional file 2: Table S2. Risk of bias assessment of randomized controlled trials. Table S3. Risk of bias assessment of observational studies. (DOCX $20 \mathrm{~kb}$ )

Additional file 3: Table S4. Inconsistency assessment. (DOCX 14 kb) Additional file 4: Table S5. Results of sensitivity analyses. (DOCX $17 \mathrm{~kb}$ )

\section{Abbreviations}

AB: Acinetobacter baumannii; APACHE II: Acute Physiology and Chronic Health Evaluation II; CAR: Carbapenem; Cl: Confidence interval; COL: Colistin; CR: Carbapenem-resistant; Crl: Credible interval; DIC: Deviance information criterion; FOS: Fosfomycin; GLY: Glycopeptide; HAP: Hospital-acquired pneumonia; HD: High dose; IH: Inhaled; IMI: Imipenem; IV: Intravenous; KP: Klebsiella pneumoniae; MCMC: Markov chain Monte Carlo;

MDR: Multidrug-resistant; MERO: Meropenem; MIU: Million international units; $\mathrm{n} / \mathrm{N}$ : Number of outcome events/number of patients in each intervention; NR: Not reported; OR: Odds ratio; PA: Pseudomonas aeruginosa; PRISMA: Preferred Reporting Items for Systematic Reviews and Meta-analyses; RIF: Rifampin; SAPS: Simplified acute physiology score; SOFA: Sepsis-related organ failure assessment score; SUCRA: Surface under the cumulative ranking curve; SUL: Sulbactam; TDM: Therapeutic drug monitoring; TEl: Teicoplanin; TIG: Tigecycline; VAN: Vancomycin; VAP: Ventilator-associated pneumonia; XDR: Extensively drug-resistant

\section{Acknowledgements}

This study was supported by the Research Institutes of Pharmaceutical Science in Seoul National University.

\section{Funding}

No funding was received for this analysis.

\section{Availability of data and materials}

All data supporting the results and conclusions reported in this article are included in this published article.

\section{Authors' contributions}

SYJ, SHL, HN, and SY searched the literature, reviewed the articles, and performed the quality assessment. SYJ and SYL participated in statistical analyses and data interpretation. SYJ, SHL, and SYL designed the study and drafted the manuscript. JIL and EKC conceived the study, were in charge of overall direction and planning, and edited the drafted manuscript. All authors have read and approved the final manuscript.

\section{Author's information}

Not applicable.

Ethics approval and consent to participate Not applicable.

\section{Consent for publication}

Not applicable.

\section{Competing interests}

The authors declare that they have no competing interests.

\section{Publisher's Note}

Springer Nature remains neutral with regard to jurisdictional claims in published maps and institutional affiliations.

\section{Author details}

'Department of Pharmacy, College of Pharmacy, Seoul National University, 1 Gwanak-ro, Gwanak-gu, Seoul 08826, Republic of Korea. ${ }^{2}$ Research Institute of Pharmaceutical Sciences, Seoul National University, Seoul, Republic of Korea. ${ }^{3}$ Department of Pharmacy, College of Pharmacy, Kyung Hee University, 26 Kyungheedae-ro, Dongdaemun-gu, Seoul 02447, Republic of Korea. ${ }^{4}$ Department of Public Health Science, Graduate School of Public Health, Seoul National University, Seoul, Republic of Korea. ${ }^{5}$ Department of Pharmacy, College of Pharmacy, Yonsei University, Incheon, Republic of Korea.

Received: 27 September 2017 Accepted: 4 December 2017

Published online: 20 December 2017

\section{References}

1. Joseph NM, Sistla S, Dutta TK, Badhe AS, Parija SC. Ventilator-associated pneumonia: a review. Eur J Intern Med. 2010;21:360-8.

2. Kalanuria AA, Zai W, Mirski M. Ventilator-associated pneumonia in the ICU. Crit Care. 2014;18:208.

3. Inchai J, Pothirat C, Bumroongkit C, Limsukon A, Khositsakulchai W, Liwsrisakun C. Prognostic factors associated with mortality of drug-resistant Acinetobacter baumannii ventilator-associated pneumonia. J Intensive Care. 2015;3:9.

4. Chastre J, Fagon JY. Ventilator-associated pneumonia. Am J Respir Crit Care Med. 2002;165:867-903.

5. Zilberberg MD, Nathanson BH, Sulham K, Fan W, Shorr AF. Multidrug resistance, inappropriate empiric therapy, and hospital mortality in Acinetobacter baumannii pneumonia and sepsis. Crit Care. 2016;20:221.

6. Sheng W-H, Liao C-H, Lauderdale T-L, Ko W-C, Chen Y-S, Liu J-W, Lau Y-J, Wang L-S, Liu K-S, Tsai T-Y. A multicenter study of risk factors and outcome of hospitalized patients with infections due to carbapenem-resistant Acinetobacter baumannii. Int J Infect Dis. 2010;14:e764-9.

7. Huttner B, Jones M, Rubin MA, Neuhauser MM, Gundlapalli A, Samore M. Drugs of last resort? The use of polymyxins and tigecycline at US Veterans Affairs medical centers, 2005-2010. PLoS One. 2012;7:e36649.

8. Viehman JA, Nguyen MH, Doi Y. Treatment options for carbapenemresistant and extensively drug-resistant Acinetobacter baumannii infections. Drugs. 2014;74:1315-33.

9. Chu H, Zhao L, Wang M, Liu Y, Gui T, Zhang J. Sulbactam-based therapy for Acinetobacter baumannii infection: a systematic review and meta-analysis. Braz J Infect Dis. 2013;17:389-94.

10. Garnacho-Montero J, Dimopoulos G, Poulakou G, Akova M, Cisneros JM, De Waele J, Petrosillo N, Seifert H, Timsit JF, Vila J, et al. Task force on management and prevention of Acinetobacter baumannii infections in the ICU. Intensive Care Med. 2015;41:2057-75.

11. Peleg AY, Hooper DC. Hospital-acquired infections due to gram-negative bacteria. N Engl J Med. 2010;362:1804-13.

12. Shen $F$, Han $Q$, Xie D, Fang $M$, Zeng $H$, Deng Y. Efficacy and safety of tigecycline for the treatment of severe infectious diseases: an updated meta-analysis of RCTs. Int J Infect Dis. 2015;39:25-33.

13. Zampieri FG, Nassar Jr AP, Gusmao-Flores D, Taniquchi LU, Torres A, Ranzani OT. Nebulized antibiotics for ventilator-associated pneumonia: a systematic review and meta-analysis. Crit Care. 2015;19:150.

14. Gu W-J, Wang F, Tang L, Bakker J, Liu J-C. Colistin for the treatment of ventilator-associated pneumonia caused by multidrug-resistant Gramnegative bacteria: a systematic review and meta-analysis. Int J Antimicrob Agents. 2014;44:477-85.

15. Liu D, Zhang J, Liu H-X, Zhu Y-G, Qu J-M. Intravenous combined with aerosolised polymyxin versus intravenous polymyxin alone in the treatment of pneumonia caused by multidrug-resistant pathogens: a systematic review and meta-analysis. Int J Antimicrob Agents. 2015;46:603-9.

16. Ni W, Han Y, Zhao J, Wei C, Cui J, Wang R, Liu Y. Tigecycline treatment experience against multidrug-resistant Acinetobacter baumannii infections: 
a systematic review and meta-analysis. Int J Antimicrob Agents. 2016;47: 107-16.

17. Jansen JP, Trikalinos T, Cappelleri JC, Daw J, Andes S, Eldessouki R, Salanti G. Indirect treatment comparison/network meta-analysis study questionnaire to assess relevance and credibility to inform health care decision making: an ISPORAMCP-NPC Good Practice Task Force report. Value Health. 2014;17:157-73.

18. Li T, Puhan MA, Vedula SS, Singh S, Dickersin K. Network meta-analysishighly attractive but more methodological research is needed. BMC Med. 2011;9:79.

19. Sutton AJ, Abrams KR. Bayesian methods in meta-analysis and evidence synthesis. Stat Methods Med Res. 2001;10:277-303.

20. Ahn J, Choi SM, Cho S, Tchoe HJ. Bayesian meta-analysis method (English version). 1st ed. Seoul: National Evidence-based Healthcare Collaborating Agency (NECA); 2014:14.

21. Hutton B, Salanti G, Caldwell DM, Chaimani A, Schmid CH, Cameron C, loannidis JP, Straus S, Thorlund K, Jansen JP. The PRISMA extension statement for reporting of systematic reviews incorporating network metaanalyses of health care interventions: checklist and explanations. Ann Intern Med. 2015;162:777-84.

22. Higgins JP, Altman DG, Gotzsche PC, Juni P, Moher D, Oxman AD, Savovic J, Schulz KF, Weeks L, Sterne JA. The Cochrane Collaboration's tool for assessing risk of bias in randomised trials. BMJ. 2011;343:d5928.

23. Wells G, Shea B, O'connell D, Peterson J, Welch V, Losos M, Tugwell P. The Newcastle-Ottawa Scale (NOS) for assessing the quality of nonrandomised studies in meta-analyses. http://www.ohri.ca/programs/clinical_ epidemiology/oxford.asp. Accessed 21 Mar 2017.

24. Yang Y, Zhang F, Skrip L, Lei H, Luo S, Lu K, Hu D. Lack of an association between angiotensin receptor blocker based therapy and increased risk of cancer: evidence from large observational studies. PLoS One. 2015;10:e0119775.

25. Zhang YP, Wan YD, Sun YL, Li J, Zhu RT. Aspirin might reduce the incidence of pancreatic cancer: a meta-analysis of observational studies. Sci Rep. 2015; 5:15460.

26. Betrosian AP, Frantzeskaki F, Xanthaki A, Georgiadis G. High-dose ampicillinsulbactam as an alternative treatment of late-onset VAP from multidrugresistant Acinetobacter baumannii. Scand J Infect Dis. 2007;39:38-43.

27. Garnacho-Montero J, Ferrandiz-Millon C. High dose of tigecycline for extremely resistant Gram-negative pneumonia: yes, we can. Crit Care. 2014; 18:157.

28. Nation RL, Garonzik SM, Li J, Thamlikitkul V, Giamarellos-Bourboulis EJ, Paterson DL, Turnidge JD, Forrest A, Silveira FP. Updated US and European dose recommendations for intravenous colistin: how do they perform? Clin Infect Dis. 2016:62:552-8.

29. Donegan S, Williamson P, D'Alessandro U, Tudur SC. Assessing key assumptions of network meta-analysis: a review of methods. Res Synth Methods. 2013:4:291-323.

30. Higgins JP, Green S. Cochrane handbook for systematic reviews of interventions. In: Deeks JJ, Higgins JP and Altman DG, editors. Heterogeneity. Chichester: Wiley; 2008. p. 276-81.

31. Jansen JP, Naci H. Is network meta-analysis as valid as standard pairwise meta-analysis? It all depends on the distribution of effect modifiers. BMC Med. 2013;11(1).

32. Higgins J, Jackson D, Barrett J, Lu G, Ades A, White I. Consistency and inconsistency in network meta-analysis: concepts and models for multi-arm studies. Res Synth Methods. 2012;3:98-110.

33. Dias S, Welton NJ, Sutton AJ, Caldwell DM, Lu G, Ades A. Evidence synthesis for decision making 4 inconsistency in networks of evidence based on randomized controlled trials. Med Decis Making. 2013;33:641-56.

34. Jackson D, Barrett JK, Rice S, White IR, Higgins J. A design-by-treatment interaction model for network meta-analysis with random inconsistency effects. Stat Med. 2014;33:3639-54.

35. Spiegelhalter DJ, Best NG, Carlin BP, Van Der Linde A. Bayesian measures of model complexity and fit. J R Stat Soc Ser B (Statistical Methodology). 2002; 64:583-639.

36. Gelman A, Rubin DB. Markov chain Monte Carlo methods in biostatistics. Stat Methods Med Res. 1996;5:339-55.

37. Salanti G, Ades A, loannidis JP. Graphical methods and numerical summaries for presenting results from multiple-treatment meta-analysis: an overview and tutorial. J Clin Epidemiol. 2011;64:163-71.

38. Connor JT, Elm JJ, Broglio KR. Bayesian adaptive trials offer advantages in comparative effectiveness trials: an example in status epilepticus. J Clin Epidemiol. 2013;66:S130-7.
39. Cope S, Donohue JF, Jansen JP, Kraemer M, Capkun-Niggli G, Baldwin M, Buckley F, Ellis A, Jones P. Comparative efficacy of long-acting bronchodilators for COPD: a network meta-analysis. Respir Res. 2013;14:100.

40. Pacou M, Taieb V, Belhadi D, Mesana L, Gauthier A. Interpreting results from Bayesian network meta-analyses (NMA): a guide for non-statisticians. Value Health. 2015;18:A718-719.

41. Burton PR, Gurrin LC, Campbell MJ. Clinical significance not statistical significance: a simple Bayesian alternative to p values. J Epidemiol Community Health. 1998;52:318-23.

42. Gurrin LC, Kurinczuk JJ, Burton PR. Bayesian statistics in medical research: an intuitive alternative to conventional data analysis. J Eval Clin Pract. 2000;6: 193-204.

43. Peter JV, John P, Graham PL, Moran JL, George IA, Bersten A. Corticosteroids in the prevention and treatment of acute respiratory distress syndrome (ARDS) in adults: meta-analysis. BMJ. 2008:336:1006-9.

44. Abdellatif S, Trifi A, Daly F, Mahjoub K, Nasri R, Lakhal SB. Efficacy and toxicity of aerosolised colistin in ventilator-associated pneumonia: a prospective, randomised trial. Ann Intensive Care. 2016;6:1.

45. Aydemir H, Akduman D, Piskin N, Comert F, Horuz E, Terzi A, Kokturk F, Ornek T, Celebi G. Colistin vs. the combination of colistin and rifampicin for the treatment of carbapenem-resistant Acinetobacter baumannii ventilatorassociated pneumonia. Epidemiol Infect. 2013;141:1214-22.

46. Durante-Mangoni E, Signoriello G, Andini R, Mattei A, Cristoforo M, Murino P, Bassetti M, Malacarne P, Petrosillo N, Galdieri N, et al. Colistin and rifampicin compared with colistin alone for the treatment of serious infections due to extensively drug-resistant Acinetobacter baumannii: a multicenter, randomized clinical trial. Clin Infect Dis. 2013;57:349-58.

47. Sirijatuphat $R$, Thamlikitkul V. Preliminary study of colistin versus colistin plus fosfomycin for treatment of carbapenem-resistant Acinetobacter baumannii infections. Antimicrob Agents Chemother. 2014;58:5598-601.

48. Amin M, Rashad A, Fouad A, Azeem AA. Re-emerging of colistin for treatment of nosocomial pneumonia due to Gram negative multi-drug resistant pathogens in critically ill patients. Egypt J Chest Dis Tuberc. 2013; 62:447-51.

49. Betrosian AP, Frantzeskaki F, Xanthaki A, Douzinas EE. Efficacy and safety of high-dose ampicillin/sulbactam vs. colistin as monotherapy for the treatment of multidrug resistant Acinetobacter baumannii ventilatorassociated pneumonia. J Infect. 2008;56:432-6.

50. Frantzeskaki F, Karampi ES, Papadopoulos P, Paramythiotou $E_{,}$ Theodorakopoulou M, Flevari A, Rizos M, Diakaki C, Armaganidis A, Dimopoulos G. Comparative study of the combination of high doses of ampicilline/sulbactam with colistin versus meropenem with colistin in ventilatior associated pneumonia caused by Acinetobacter baumannii. Intensive Care Med. 2013;39(Suppl 2):S383.

51. Chuang YC, Cheng CY, Sheng WH, Sun HY, Wang JT, Chen YC, Chang SC. Effectiveness of tigecycline-based versus colistin-based therapy for treatment of pneumonia caused by multidrug-resistant Acinetobacter baumannii in a critical setting: a matched cohort analysis. BMC Infect Dis. 2014;14:102.

52. De Pascale G, Montini L, Pennisi M, Bernini V, Maviglia R, Bello G, Spanu T, Tumbarello M, Antonelli M. High dose tigecycline in critically ill patients with severe infections due to multidrug-resistant bacteria. Crit Care. 2014;18:R90.

53. Demirdal T, Sari US, Nemli SA. Is inhaled colistin beneficial in ventilator associated pneumonia or nosocomial pneumonia caused by Acinetobacter baumannii? Ann Clin Microbiol Antimicrob. 2016;15:1.

54. Doshi NM, Cook CH, Mount KL, Stawicki SP, Frazee EN, Personett HA, Schramm GE, Arnold HM, Murphy CV. Adjunctive aerosolized colistin for multi-drug resistant gram-negative pneumonia in the critically ill: a retrospective study. BMC Anesthesiol. 2013;13:45.

55. Garnacho-Montero J, Amaya-Villar R, Gutierrez-Pizarraya A, Espejo-Gutierrez DTE, Artero-Gonzalez ML, Corcia-Palomo Y, Bautista-Paloma J. Clinical efficacy and safety of the combination of colistin plus vancomycin for the treatment of severe infections caused by carbapenem-resistant Acinetobacter baumannii. Chemotherapy. 2013;59:31.

56. Hsieh TC, Chen FL, Ou TY, Jean SS, Lee WS. Role of aerosolized colistin methanesulfonate therapy for extensively-drug-resistant Acinetobacter baumannii complex pneumonia and airway colonization. J Microbiol Immunol Infect. 2016;49:523-30.

57. Kalin G, Alp E, Coskun R, Demiraslan H, Gündogan K, Doganay M. Use of high-dose IV and aerosolized colistin for the treatment of multidrug- 
resistant Acinetobacter baumannii ventilator-associated pneumonia: do we really need this treatment? J Infect Chemother. 2012;18:872-7.

58. Khawcharoenporn T, Pruetpongpun N, Tiamsak P, Rutchanawech S, Mundy LM, Apisarnthanarak A. Colistin-based treatment for extensively drugresistant Acinetobacter baumannii pneumonia. Int J Antimicrob Agents. 2014:43:378-82.

59. Kim WY, Moon JY, Huh JW, Choi SH, Lim CM, Koh Y, Chong YP, Hong SB. Comparable efficacy of tigecycline versus colistin therapy for multidrugresistant and extensively drug-resistant Acinetobacter baumannii pneumonia in critically ill patients. PLoS One. 2016;11:e0150642.

60. Kofteridis DP, Alexopoulou C, Valachis A, Maraki S, Dimopoulou D, Georgopoulos D, Samonis G. Aerosolized plus intravenous colistin versus intravenous colistin alone for the treatment of ventilator-associated pneumonia: a matched case-control study. Clin Infect Dis. 2010;51:1238-44

61. Korbila IP, Michalopoulos A, Rafailidis PI, Nikita D, Samonis G, Falagas ME. Inhaled colistin as adjunctive therapy to intravenous colistin for the treatment of microbiologically documented ventilator-associated pneumonia: a comparative cohort study. Clin Microbiol Infect. 2010;16: 1230-6

62. Kwon SH, Ahn HL, Han OY, La HO. Efficacy and safety profile comparison of colistin and tigecycline on the extensively drug resistant Acinetobacter baumannii. Biol Pharm Bull. 2014;37:340-6.

63. Petrosillo N, Giannella M, Antonelli M, Antonini M, Barsic B, Belancic L, Inkaya AC, De Pascale G, Grilli E, Tumbarello M, Akova M. Clinical experience of colistin-glycopeptide combination in critically ill patients infected with Gram-negative bacteria. Antimicrob Agents Chemother. 2014;58:851-8.

64. Tumbarello M, De Pascale G, Trecarichi EM, De Martino S, Bello G, Maviglia R, Spanu T, Antonelli M. Effect of aerosolized colistin as adjunctive treatment on the outcomes of microbiologically documented ventilatorassociated pneumonia caused by colistin-only susceptible gram-negative bacteria. CHEST J. 2013;144:1768-75.

65. Yilmaz GR, Guven T, Guner R, Kocak Tufan Z, Izdes S, Tasyaran MA, Acikgoz ZC. Colistin alone or combined with sulbactam or carbapenem against A. baumannii in ventilator-associated pneumonia. J Infect Dev Ctries. 2015;9: 476-85.

66. Zalts R, Neuberger A, Hussein K, Raz-Pasteur A, Geffen Y, Mashiach T, Finkelstein R. Treatment of carbapenem-resistant Acinetobacter baumannii ventilator-associated pneumonia: retrospective comparison between intravenous colistin and intravenous ampicillin-sulbactam. Am J Ther. 2016; 23:e78-85.

67. Lin HS, Lee MH, Cheng CW, Hsu PC, Leu HS, Huang CT, Ye JJ. Sulbactam treatment for pneumonia involving multidrug-resistant Acinetobacter calcoaceticus-Acinetobacter baumannii complex. Infect Dis (Lond). 2015; 47(6):370-8

68. Busey K, Ferreira J, Aldridge P, Johnson D, Guzman N, Jankowski CA. Treatment efficacy of ampicillin/sulbactam in comparison to alternative beta-lactams for severe Acinetobacter baumannii infections. Infect Dis. 2016; 48:775-7.

69. Ye J-J, Lin H-S, Yeh C-F, Wu Y-M, Huang P-Y, Yang C-C, Huang C-T, Lee M-H. Tigecycline-based versus sulbactam-based treatment for pneumonia involving multidrug-resistant Acinetobacter calcoaceticus-Acinetobacter baumannii complex. BMC Infect Dis. 2016;16:374.

70. Oliveira M, Prado G, Costa S, Grinbaum R, Levin A. Ampicillin/sulbactam compared with polymyxins for the treatment of infections caused by carbapenem-resistant Acinetobacter spp. J Antimicrob Chemother. 2008;61: 1369-75.

71. Jaruratanasirikul S, Wongpoowarak W, Aeinlang N, Jullangkoon M. Pharmacodynamics modeling to optimize dosage regimens of sulbactam. Antimicrob Agents Chemother. 2013;57:3441-4.

72. Adnan S, Paterson DL, Lipman J, Roberts JA. Ampicillin/sulbactam: its potential use in treating infections in critically ill patients. Int J Antimicrob Agents. 2013;42:384-9.

73. Hoosen A, Baba K. In-vitro activity of fosfomycin against clinical isolates of carbapenem resistant Acinetobacter baumannii complex and Pseudomonas aeruginosa at a South African academic hospital. Int J Infect Dis. 2016; 45(Suppl 1):92.

74. Pontikis K, Karaiskos I, Bastani S, Dimopoulos G, Kalogirou M, Katsiari M, Oikonomou A, Poulakou G, Roilides E, Giamarellou H. Outcomes of critically ill intensive care unit patients treated with fosfomycin for infections due to pandrug-resistant and extensively drug-resistant carbapenemase-producing Gram-negative bacteria. Int J Antimicrob Agents. 2014;43:52-9.
75. Karageorgopoulos DE, Wang R. Yu X-h, Falagas ME: Fosfomycin: evaluation of the published evidence on the emergence of antimicrobial resistance in Gram-negative pathogens. J Antimicrob Chemother. 2011;67:255-68.

76. Kalil AC, Metersky ML, Klompas M, Muscedere J, Sweeney DA, Palmer LB, Napolitano LM, O'Grady NP, Bartlett JG, Carratalà J, et al. Management of adults with hospital-acquired and ventilator-associated pneumonia: 2016 clinical practice guidelines by the Infectious Diseases Society of America and the American Thoracic Society. Clin Infect Dis. 2016;63:e61-e111.

77. Arnold HM, Sawyer AM, Kollef MH. Use of adjunctive aerosolized antimicrobial therapy in the treatment of Pseudomonas aeruginosa and Acinetobacter baumannii ventilator-associated pneumonia. Respir Care. 2012;57:1226-33.

78. Kollef MH, Ricard JD, Roux D, Francois B, Ischaki E, Rozgonyi Z, Boulain T, Ivanyi Z, Janos G, Garot D, et al. A Randomized Trial of the Amikacin Fosfomycin Inhalation System for the Adjunctive Therapy of Gram-Negative Ventilator-Associated Pneumonia: IASIS Trial. Chest. 2017;151:1239-46.

79. Niederman MS, Chastre J, Corkery K, Fink JB, Luyt CE, Garcia MS. BAY41-6551 achieves bactericidal tracheal aspirate amikacin concentrations in mechanically ventilated patients with Gram-negative pneumonia. Intensive Care Med. 2012;38:263-71.

80. Ghannam DE, Rodriguez GH, Raad II, Safdar A. Inhaled aminoglycosides in cancer patients with ventilator-associated Gram-negative bacterial pneumonia: safety and feasibility in the era of escalating drug resistance. Eur J Clin Microbiol Infect Dis. 2009;28:253-9.

81. Michalopoulos AS, Falagas ME. Inhaled antibiotics in mechanically ventilated patients. Minerva Anestesiol. 2014:80:236-44.

82. Wright $\mathrm{H}$, Bonomo RA, Paterson DL. New agents for the treatment of infections with Gram-negative bacteria: restoring the miracle or false dawn? Clin Microbiol Infect. 2017;23:704-12.

83. Solomkin JS, Ramesh MK, Cesnauskas G, Novikovs N, Stefanova P, Sutcliffe JA, Walpole SM, Horn PT. Phase 2, randomized, double-blind study of the efficacy and safety of two dose regimens of eravacycline versus ertapenem for adult community-acquired complicated intra-abdominal infections. Antimicrob Agents Chemother. 2014;58:1847-54.

84. Falagas ME, Mavroudis AD, Vardakas KZ. The antibiotic pipeline for multidrug resistant gram negative bacteria: what can we expect? Expert Rev Anti Infect Ther. 2016;14:747-63.

\section{Submit your next manuscript to BioMed Central and we will help you at every step:}

- We accept pre-submission inquiries

- Our selector tool helps you to find the most relevant journal

- We provide round the clock customer support

- Convenient online submission

- Thorough peer review

- Inclusion in PubMed and all major indexing services

- Maximum visibility for your research

Submit your manuscript at www.biomedcentral.com/submit
C Biomed Central 